\title{
Ischemic Preconditioning in Chronically Hypoxic Neonatal Rat Heart
}

\author{
IVANA OŠTÁDALOVÁ, BOHUSLAV OŠTÁDAL, DANIELA JARKOVSKÁ, AND \\ FRANTIŠEK KOLÁR̆ \\ Institute of Physiology, Academy of Sciences of the Czech Republic, Center for Experimental \\ Cardiovascular Research, Prague, Czech Republic [I.O., B.O., F.K.]; Institute of Histology and \\ Embryology, Faculty of Medicine, Charles University, Prague, Czech Republic [D.J.]
}

\begin{abstract}
ABST
Rat hearts isolated on $\mathrm{d} 1,4,7$, and 10 of postnatal life were
perfused (in Langendorff mode) with Krebs-Henseleit solution at
constant pressure, temperature, and stimulation rate. Recovery of
the contractile function after global ischemia was measured by an
isometric force transducer and analyzed using an online com-
puter. Ischemic preconditioning (IP) was induced by three 3 -min
periods of global ischemia, each separated by a 5 -min period of
reperfusion. Prenatal hypoxia was induced by exposure of preg-
nant mothers to intermittent high altitude (IHA), simulated in a
barochamber ( 8 h/d, $5000 \mathrm{~m})$ from $\mathrm{d} 15$ to 20 of pregnancy.
Postnatal hypoxia was simulated by the identical procedure from
postnatal $\mathrm{d} 1$ to 6 and 9. Prenatal exposure to IHA failed to
improve ischemic tolerance on $\mathrm{d} 1$, but postnatal exposure to
IHA improved recovery of the developed force after ischemia on
$\mathrm{d} 7$ (33 $\pm 3 \%$ versus $43 \pm 4 \%$ and 10 ( $39 \pm 2 \%$ versus $54 \pm$
$2 \%$ ). Combination of IHA and IP induced higher protective
effects in all age groups, including postnatal $\mathrm{d} 1$ ( $48 \pm 2 \%$ versus
$56 \pm 3 \%$ ), whereas IHA and IP applied separately failed to
improve ischemic tolerance. Neither the mitochondrial $\mathrm{K}_{\mathrm{ATP}}$
\end{abstract}
\section{ABSTRACT}

channel blocker 5-hydroxydecanoate nor the nitric oxide synthase inhibitor $N \omega$-nitro-L-arginine methyl ester abolished protection by IP in normoxic animals, but they decreased significantly protection by IHA hypoxia. The final recovery was even lower than the corresponding normoxic values. It seems likely that mitochondrial $\mathrm{K}_{\mathrm{ATP}}$ channels and nitric oxide may be involved in the protective mechanisms of adaptation to chronic hypoxia but not to that of IP, at least in neonates. (Pediatr Res 52: 561-567, 2002)
DF, developed force

\section{Abbreviations}
5-HD, 5-hydroxydecanoate
L-NAME, $N \omega$-nitro-L-arginine methyl ester
IHA, intermittent high altitude
$\mathbf{N}$, normoxic
$\mathbf{H}$, hypoxic
I, ischemia
IP, ischemic preconditioning

The degree of hypoxic injury depends not only on the intensity and duration of the hypoxic stimulus but also on the level of cardiac tolerance to oxygen deprivation. This parameter changes significantly during ontogenetic development, and the time course is different in males and females. The immature mammalian heart is more resistant to oxygen deprivation than that of the adult, but the mechanisms of this difference have not yet been satisfactorily clarified (for review, see Ref. 1). The interest of many experimental and clinical cardiologists during the past $40 \mathrm{y}$ has been focused on the question of how cardiac tolerance to oxygen deprivation might be increased. In this regard, the two most potent protective mechanisms have been

Received November 19, 2001; accepted April 2, 2002.

Correspondence: Ivana Oštádalová, Institute of Physiology, Academy of Sciences of the Czech Republic, Videňská 1083, 14220 Prague 4, Czech Republic; e-mail: iostadal@biomed.cas.cz

Supported by grant No. 305/00/1659 from the Grant Agency of the Czech Republic.

DOI: 10.1203/01.PDR.0000030879.20888.5B described: long-lasting adaptation to chronic hypoxia $(2,3)$, and short-lasting adaptation, called "ischemic preconditioning" (4).

Whereas a substantial amount of data are available concerning protection of the adult myocardium, information on whether these protective phenomena also occur in immature heart is inadequate (1). We have observed (5) that chronic hypoxia, simulated in a barochamber, results in similarly enhanced cardiac resistance (expressed as the recovery of contractile function of the isolated right ventricle after acute anoxia in vitro) in rats exposed to chronic hypoxia for $5 \mathrm{wk}$ either from d 4 of postnatal life or in adulthood. Furthermore, Baker et al. (6) demonstrated that postnatal adaptation to chronic hypoxia (from d 7 to 28 of postnatal life) increased the tolerance of the developing rabbit heart: postischemic recovery of aortic flow at these stages was better in chronically hypoxic hearts than in age-matched controls. Unfortunately, no data on the effect of prenatal and/or early postnatal exposure to a hypoxic environment on cardiac tolerance of neonates are 
available. As far as the IP is concerned, we have shown $(7,8)$ that this phenomenon is absent in rats at birth and that the enhanced postischemic recovery of the contractile function only develops during the second postnatal week. Our results were confirmed by Awad et al. (9); they have shown that the absence of preconditioning in immature isolated hearts cannot be overdriven by increasing the preconditioning stimulus. Liu et al. (10) and Baker et al. (11) observed that preconditioning can be induced in isolated perfused immature rabbit hearts; data on the newborns are, however, lacking.

The precise mechanisms of cardioprotection by adaptation to chronic hypoxia and IP in the adult myocardium are still unclear and the same is true for immature heart (for review, see Refs. 12, 13). Recently, it has been shown that long-term adaptation to chronic hypoxia results in enhanced activation of mitochondrial $\mathrm{K}_{\text {ATP }}$ channels in the heart of adult rats (14) as well as in the myocardium of immature rabbits (15). Similarly, increasing evidence has accumulated in support of mitochondrial $\mathrm{K}_{\text {АTP }}$ channels as a trigger, mediator, and effector of cardioprotection by IP, again both in adult (16) and immature (11) rabbit hearts. Furthermore, mediators derived from the endothelium, particularly nitric oxide (NO), have been suggested to play a role in the cardioprotective effect of preconditioning in adult dogs (17) as well as of adaptation to chronic hypoxia in immature rabbits (18).

In this connection, the question arises whether ischemic tolerance of the heart adapted to chronic hypoxia can be further increased by IP and whether both cardioprotective phenomena share similar pathways, at least in part. Literary data are scarce and rather controversial $(11,14,19,20)$. Data on newborn animals as well as on the possible effeet of prenatal exposure to hypoxia are not available. The clinical relevance of this question is obvious: many children undergoing eardiac surgery in the first year of life exhibit varying degrees of cyanotic heart disease where the myocardium is chronically perfused with hypoxic blood. Understanding the pathways by which cyanotic congenital heart disease modifies the protective mechanisms during ischemia may, for example, provide insight into developing treatments for limiting myocardial damage during surgery (21).

We have shown previously that the neonatal period represents in the rat myocardium the period of dramatic changes in cardiac contractile performance, inotropic responsiveness, and tolerance to hypoxic deprivation $(8,22,23)$. The aim of the present study was, therefore, 1) to answer the question whether the already high ischemic tolerance of the neonatal rat heart can be further increased by i) prenatal or early postnatal exposure to IHA hypoxia alone or ii) by combination of IHA and IP; and 2) to analyze the possible role of mitochondrial $\mathrm{K}_{\mathrm{ATP}}$ channels and NO in the protection of neonatal heart.

\section{METHODS}

All the investigations conformed with the National Institutes of Health Guide for the Care and Use of Laboratory Animals.

Animal model. A total of 248 neonatal male Wistar rats were used throughout the experiments. They were studied at the ages of $1,4,7$, and $10 \mathrm{~d}$ of postnatal life. The group of 1-d-old newborns was exposed to hypoxia prenatally: their mothers were acclimatized to IHA hypoxia simulated in a hypobaric chamber (altitude of $5000 \mathrm{~m}$, barometric pressure $\left.405 \mathrm{~mm} \mathrm{Hg}, 54 \mathrm{kPa}, \mathrm{Po}_{2}=85 \mathrm{~mm} \mathrm{Hg}, 11.3 \mathrm{kPa}\right) 8 \mathrm{~h} / \mathrm{d}$, from d 15 to 20 of pregnancy (total of five exposures). Animals in the older age groups, i.e. 7- and 10-d-old neonates, were exposed to the same IHA in nests together with their mothers starting from the first day of postnatal life. The last hypoxic exposure took place $24 \mathrm{~h}$ before examination, i.e. on d 6 and 9 , respectively. Thus, the total number of exposures was either six (7-d-old) or nine (10-d-old). The control (normoxic) groups of animals were kept for the corresponding period at barometric pressure and $\mathrm{PO}_{2}$ equivalent to an altitude of $200 \mathrm{~m}$. The group of 4-d-old animals was used only for assessment of cardiac tolerance to ischemia in normoxic animals to obtain more detailed information on the time course of this parameter during the early postnatal period. All mothers had free access to water and a standard laboratory diet.

Assessment of heart function. After killing the hypoxic and normoxic animals by cervical dislocation, the chest was quickly opened and a stainless steel cannula (with an external diameter of $0.45 \mathrm{~mm}$ for $\mathrm{d} 1$ and 4 or $0.80 \mathrm{~mm}$ for $\mathrm{d} 7$ and 10) was inserted into the aorta. The heart was rapidly excised, the atria were removed, and the ventricles were perfused in the Langendorff mode under constant pressure, corresponding to the mean arterial blood pressure for each given developmental stage $(24,25)$, i.e. $25,42,57$, and $73 \mathrm{~cm} \mathrm{H}_{2} \mathrm{O}$ on $\mathrm{d} 1,4,7$, and 10 , respectively. The hearts were perfused with a KrebsHenseleit solution containing (in $\mathrm{mmol} / \mathrm{L}$ ) the following: $\mathrm{NaCl}$ 118.0, $\mathrm{KCl}$ 4.7, $\mathrm{CaCl}_{2} 1.25, \mathrm{MgSO}_{4}$ 1.2, $\mathrm{NaHCO}_{3}$ 25.0, $\mathrm{KH}_{2} \mathrm{PO}_{4}$ 1.2, glucose 7.0, and mannitol 1.1. The solution was saturated by a mixture of $95 \% \mathrm{O}_{2}$ and $5 \% \mathrm{CO}_{2}(\mathrm{pH} 7.4)$ and temperature was maintained at $37^{\circ} \mathrm{C}$. The hearts were electrically stimulated at a rate of 200 beats/min using silver electrodes attached to the base of the heart. The stimulation was performed with pulses of alternating polarity, $1 \mathrm{~ms}$ duration, and voltage set at $50 \%$ above the threshold level. The resting force was gradually inereased by means of a micromanipulator to the level at which the DF was approximately $80 \%$ of the maximum force reached at optimum preload. The contractile function of these isolated heart was measured using an isometric force transducer connected by a glass-fiber, two-arm titanium lever and silk suture ( 0.7 metric) to the apex of the heart. The DF was evaluated automatically from the force signal using an online computer $(8,22)$.

Experimental protocol. After a period of stabilization, baseline values of DF were recorded. Then, an additional 5-min perfusion was carried out and the DF was again recorded. One-half of the hearts from normoxic as well as hypoxic 1-, 7-, and 10-d-old rats were preconditioned by subjecting them to three 3-min periods of global ischemia, each separated by a 5 -min period of reperfusion. The remaining nonpreconditioned hearts from hypoxic and normoxic groups (4-d-old rats) were simply perfused during the corresponding period. All hearts were then exposed to $40 \mathrm{~min}$ of sustained global ischemia followed by reperfusion up to maximum recovery of DF [the last value of DF before its decay $(8,26)]$ (Fig. 1). DF was measured in all hearts in 3-min intervals during the reperfusion 
ISCHEMIA (I)

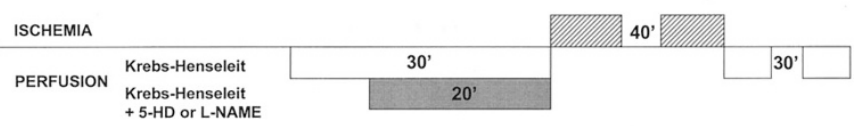

ISCHEMIA AND PRECONDITIONING $(I+\mathrm{P})$

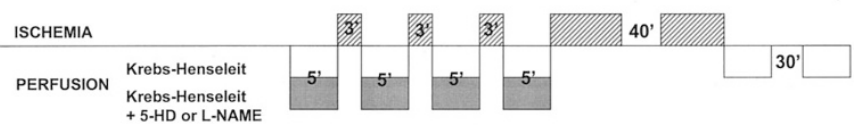

Figure 1. Experimental protocol. After a period of stabilization, the isolated hearts from the "ischemic" group (I) were perfused with Krebs-Henseleit solution with or without 5-HD and L-NAME (30 min). The hearts were then exposed to $40 \mathrm{~min}$ of sustained global ischemia followed by reperfusion ( 30 min). The hearts from the "preconditioned" group $(I+P)$ were subjected to three 3-min periods of global ischemia, each separated by a 5 -min period of reperfusion with or without 5-HD and L-NAME. The hearts were then exposed - similarly as the hearts in the ischemic group - to $40 \mathrm{~min}$ of sustained global ischemia followed by reperfusion

period. The values of DF were expressed as percentage of baseline values.

L-NAME and 5-HD. Separate groups of animals were used to examine whether the nitric oxide pathway and mitochondrial $\mathrm{K}_{\text {ATP }}$ channels are involved in the protective mechanisms of adaptation to chronic hypoxia and IP in these age groups. For this purpose, the isolated hearts from normoxic and hypoxic rats at the age of 1 or $10 \mathrm{~d}$ of postnatal life were perfused with a selective mitochondrial $\mathrm{K}_{\mathrm{ATP}}$ blocker 5-HD (300 $\mu \mathrm{M}$, Sigma RBI, St. Louis, MO, U.S.A.) or NO synthase (NOS) inhibitor L-NAME (200 $\mu$ M, Sigma Chemical, St. Louis, MO, U.S.A.). The perfusion started $5 \mathrm{~min}$ before preconditioning and finished before sustained global ischemia of $40 \mathrm{~min}$; nonpreconditioned hearts were perfused for an equivalent period of time (Fig. 1). All hearts were then subjected to reperfusion with a Krebs-Henseleit solution without drugs up to maximum recovery of DF. The values of DF were recorded as described above. After a period of stabilization, baseline values of coronary flow $(\mathrm{CF}, \mathrm{mL} / \mathrm{min} / \mathrm{g}$, measured by means of collection of the effluent) were recorded; $\mathrm{CF}$ was measured again at maximum recovery of DF during reperfusion.

Statistical analysis. The results are expressed as means \pm SEM. Each observation was obtained from at least eight heart preparations in each group. Differences in the recovery of contractile function among the groups were evaluated using, two-, and one-way ANOVA. For preliminary analysis, three way ANOVA was used. For pairwise mean comparisons, the Student-Newman-Keuls multiple range test was applied. All the programs used were from BMDP Statistical Software
(University of California). Differences were considered statistically significant when $p<0.01$.

\section{RESULTS}

Weight parameters and hematocrit. Body and heart weight parameters and hematocrit in normoxic animals and in rats exposed to IHA hypoxia are summarized in Table 1. Prenatal exposure to IHA hypoxia did not influence the body weight and hematocrit of 1-d-old animals, but significantly increased their absolute as well as relative heart weight and simultaneously decreased the percentage of heart dry substance. On the other hand, postnatal exposure to IHA hypoxia significantly decreased the body weight and absolute heart weight of 10-d-old animals, but significantly increased relative heart weight and hematocrit; the percentage of heart dry substance was decreased, similarly as in the younger group.

Tolerance of the neonatal heart to ischemia. Tolerance of the isolated rat heart, expressed as the recovery of DF after ischemia, changed significantly during the early phase of postnatal life (Fig. 2). High tolerance on postnatal d 1 is followed by a significant decrease on $\mathrm{d} 4$ and 7 ; cardiac resistance to oxygen deprivation remained lower even on d 10 as compared with d 1.

Protective effect of IP and/or IHA hypoxia. IP failed to improve recovery of DF on postnatal $\mathrm{d} 1$, but significant protective effect was observed on $\mathrm{d} 7$ and 10. Prenatal exposure to IHA hypoxia failed to increase cardiac tolerance to ischemia in 1 -d-old hearts, similarly as preconditioning. In contrast, postnatal exposure to IHA significantly improved recovery of $\mathrm{DF}$ after ischemia on $\mathrm{d} 7$ and 10; the degree of protection was similar to the effect of IP (Fig. 3).

Combination of protection by IP and IHA hypoxia. Combination of exposure to IHA hypoxia and IP induced higher protective effects as compared with both separate phenomena in all age groups under study. Surprisingly, this effect was significant even on postnatal d 1 , where both interventions applied separately failed to improve cardiac tolerance to oxygen deprivation (Fig. 3).

Effect of 5-HD and L-NAME on cardiac tolerance to ischemia. The effect of both drugs was strictly age dependent. In 1-d-old normoxic hearts, 5-HD did not influence cardiac tolerance to ischemia. On the other hand, it decreased cardiac tolerance in hypoxic animals, as the final recovery was even lower compared with normoxic animals. In the same age group, L-NAME reduced postichemic recovery already in normoxic preconditioned and nonpreconditioned hearts, and eliminated the protective effect of preconditioning in IHA-exposed rats (Fig. 4).

Table 1. Weight parameters and hematocrit in normoxic animals and in rats exposed to IHA hypoxia

\begin{tabular}{ccccccc}
\hline Rats & Age $(\mathrm{d})$ & Body weight $(\mathrm{g})$ & Heart weight $(\mathrm{mg})$ & Dry heart weight $(\%)$ & Heart/body weight $(\mathrm{mg} / \mathrm{g})$ & Hematocrit $(\%)$ \\
\hline Normoxic & 1 & $6.51 \pm 0.16$ & $26.19 \pm 0.77$ & $17.57 \pm 0.24$ & $4.03 \pm 0.08$ & $32.5 \pm 1.5$ \\
& 10 & $22.60 \pm 0.55$ & $97.12 \pm 2.80$ & $19.38 \pm 0.68$ & $4.31 \pm 0.09$ & $31.0 \pm 0.3$ \\
Hypoxic & 1 & $6.23 \pm 0.14$ & $33.31 \pm 0.46^{*}$ & $15.92 \pm 0.58^{*}$ & $5.34 \pm 0.19^{*}$ & $30.9 \pm 0.9$ \\
& 10 & $16.70 \pm 0.36^{*}$ & $88.42 \pm 2.33^{*}$ & $17.52 \pm 0.35^{*}$ & $5.35 \pm 0.15^{*}$ & $38.5 \pm 0.6^{*}$ \\
\hline
\end{tabular}

One-day-old animals were exposed to IHA hypoxia prenatally (their mothers were kept in a barochamber from d 15 to 20 of pregnancy), 10-d-old rats were exposed to IHA postnatally from d 1 to 9 ; *Significantly different $(p<0.01)$ from normoxic animals. 


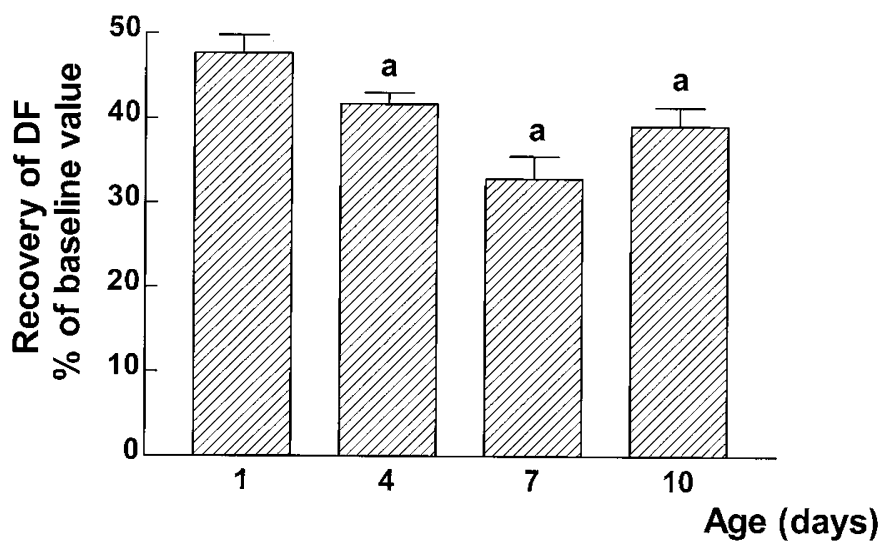

Figure 2. Tolerance of the neonatal heart to ischemia. Cardiac tolerance to ischemia (expressed as the recovery of DF after ischemia) on postnatal d 1, 4,

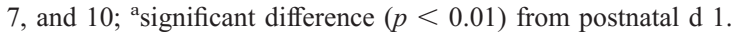

In 10-d-old hearts, neither 5-HD nor L-NAME influenced tolerance to ischemia and protection by IP in normoxic animals. They abolished protection by IHA hypoxia; the final recovery was again lower than the corresponding normoxic values. The protective effect of preconditioning in hypoxic animals was, however, preserved (Fig. 5).

Baseline values of $\mathrm{CF}(\mathrm{mL} / \mathrm{min} / \mathrm{g})$ were significantly lower in 1-d-old normoxic $(7.1 \pm 0.4)$ and hypoxic $(6.6 \pm 0.4)$ animals as compared with 10-d-old hearts (normoxic $12.6 \pm$ 1.2 , hypoxic $12.8 \pm 1.0$ ). CF measured at maximum recovery of DF during reperfusion (Table 2 ) was reducêd in all $\mathrm{L}-\mathrm{NAME}$ treated hearts. 5-HD did not influence coronary flow.

\section{DISCUSSION}

Our results have shown that cardiac tolerance to ischemia changes significantly during early phases of ontogenetic development. Detailed analysis in rat isolated hearts has confirmed our previous results $(7,8)$, showing a significant decrease of tolerance to global ischemia (expressed as postischemic recovery of DF) from d 1 to 7 . Ischemic tolerance remained lower even on d 10. Riva and Hearse (27) and Awad et al. (9) have observed that the age-dependent changes in resistance to global ischemia in the rat isolated heart showed a biphasic pattern, with increasing tolerance from the end of the first postnatal week up to the weaning period, followed by a decline to adulthood. Our results, therefore, suggest a possible triphasic pattern of the ontogenetic development of cardiac sensitivity to ischemia, with a decrease during the first week of life. The mechanisms of the higher resistance of the newborn heart to oxygen deprivation have not yet been satisfactorily clarified. As the mammalian fetus lives at oxygen partial pressure corresponding to an altitude of $8000 \mathrm{~m}$, newborn mammals exhibit a number of physiologic reactions similar to adaptative mechanisms known from hypoxia-tolerant animals, e.g. lower energy demand, greater anaerobic glycolytic capacity, ontogenetic changes in the ATP catabolic pathways, calcium handling, and sensitivity to oxygen free radicals (for review, see Ref. 1). Neonatal tolerance to oxygen deprivation seems to be primarily based on the ability to maintain tissue aerobiosis as long as possible (28).

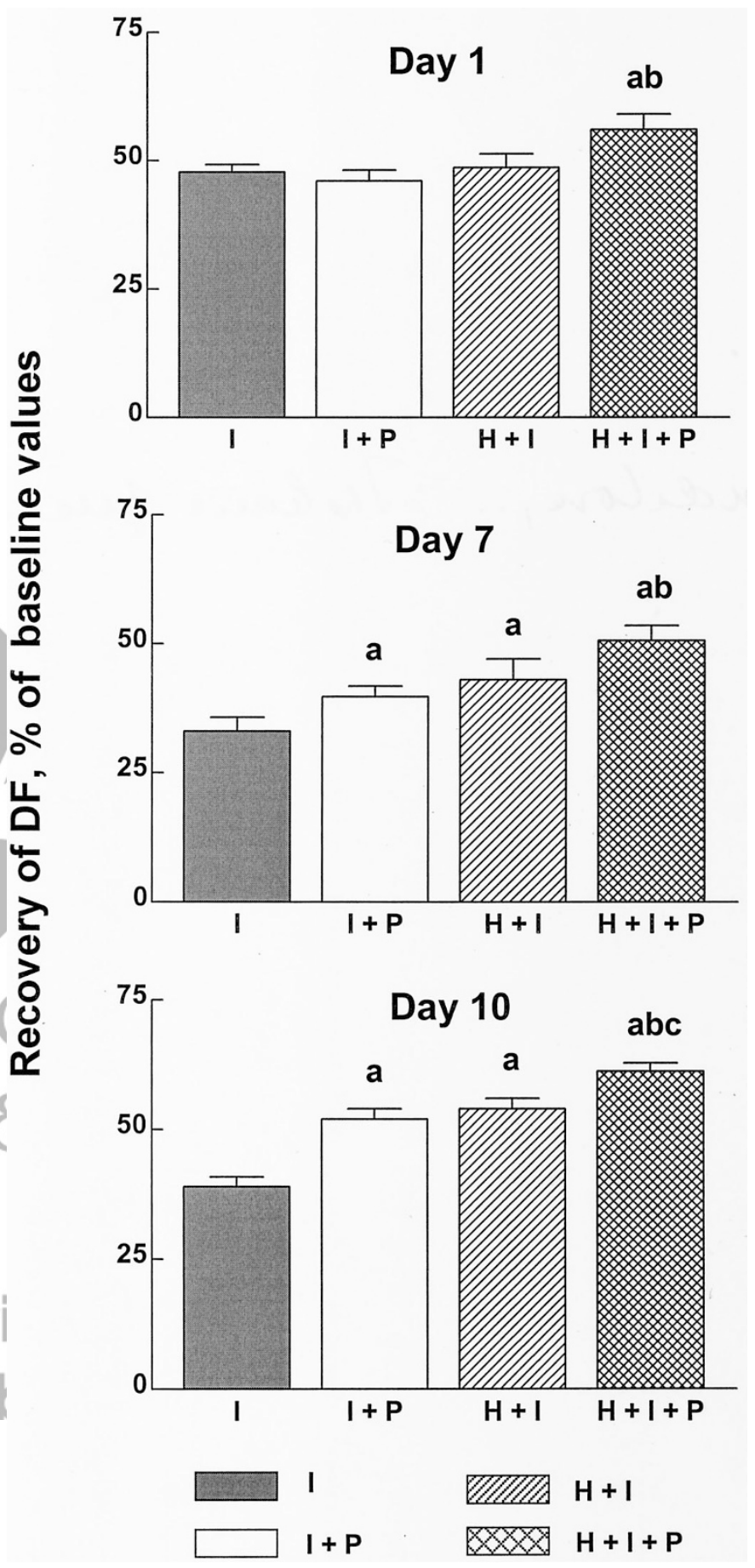

Figure 3. Combination of protection by IP and IHA hypoxia. Cardiac tolerance to ischemia (expressed as the recovery of DF after ischemia) during early phase of postnatal life in rats. Effect of ischemia (I), ischemia and preconditioning $(\mathrm{I}+\mathrm{P})$ in normoxic animals, and ischemia $(\mathrm{H}+\mathrm{I})$ and ischemia with preconditioning $(\mathrm{H}+\mathrm{I}+\mathrm{P})$ in hypoxic animals; ${ }^{\mathrm{a}}$ significant difference $(p<0.01)$ from the ischemic group (I); ${ }^{b}$ from the I + P group; ${ }^{\mathrm{c}}$ from the $\mathrm{H}+\mathrm{I}$ group.

The neonatal period seems to be critical also for the development of possibilities of cardiac protection against oxygen deprivation. We have shown that prenatal exposure to IHA hypoxia failed to increase tolerance to ischemia in 1-d-old hearts, similarly as IP in our previous study (8). In contrast, postnatal exposure to IHA significantly improved recovery of 


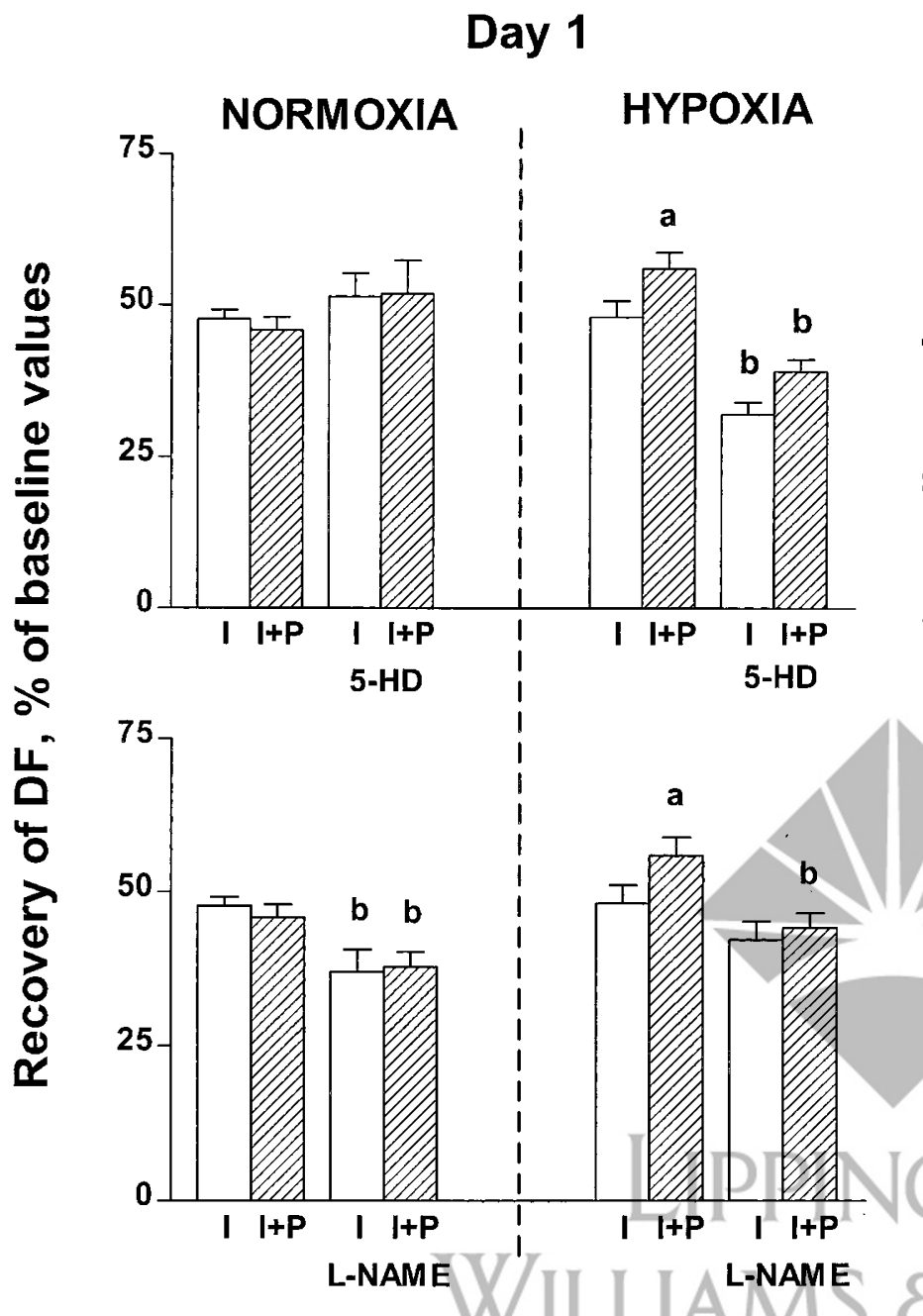

Figure 4. Effect of 5-HD and L-NAME on cardiac tolerance to ischemia, d1. Cardiac tolerance to ischemia (expressed as the recovery of DF after ischemia) on d 1 of postnatal life in rats. Effect of ischemia (I) and ischemia and preconditioning $(\mathrm{I}+\mathrm{P})$ in normoxic and hypoxic animals perfused with or without 5-HD (upper panel) and with or without L-NAME (lower panel); ${ }^{\mathrm{a}}$ significant difference $(p<0.05)$ from the ischemic group (I); ${ }^{\mathrm{b}}$ from the corresponding groups perfused without 5-HD or L-NAME.

\section{Dro 0 (o)}

DF after ischemia on $\mathrm{d} 7$ and 10, again similarly as preconditioning. It seems, therefore, that decreasing tolerance to ischemia during early postnatal life is counteracted by the development of endogenous protection; both adaptation to chronic hypoxia as well as IP alone failed to improve ischemic tolerance just after birth but their protective effects developed during the early postnatal period.

As we have shown, the protective effects of adaptation to chronic hypoxia and IP share the same developmental pattern - at least in rats during the early ontogenetic period. It was, therefore, of interest to know whether these two phenomena use the same or different pathways. We have observed that the combination of exposure to IHA hypoxia and IP induced higher protective effect as compared with both separate phenomena in all age groups under study. Surprisingly, this effect was significant even on postnatal $\mathrm{d} 1$, where both interventions applied separately failed to improve cardiac tolerance to oxygen deprivation. Data from available literature are controver-
Day 10

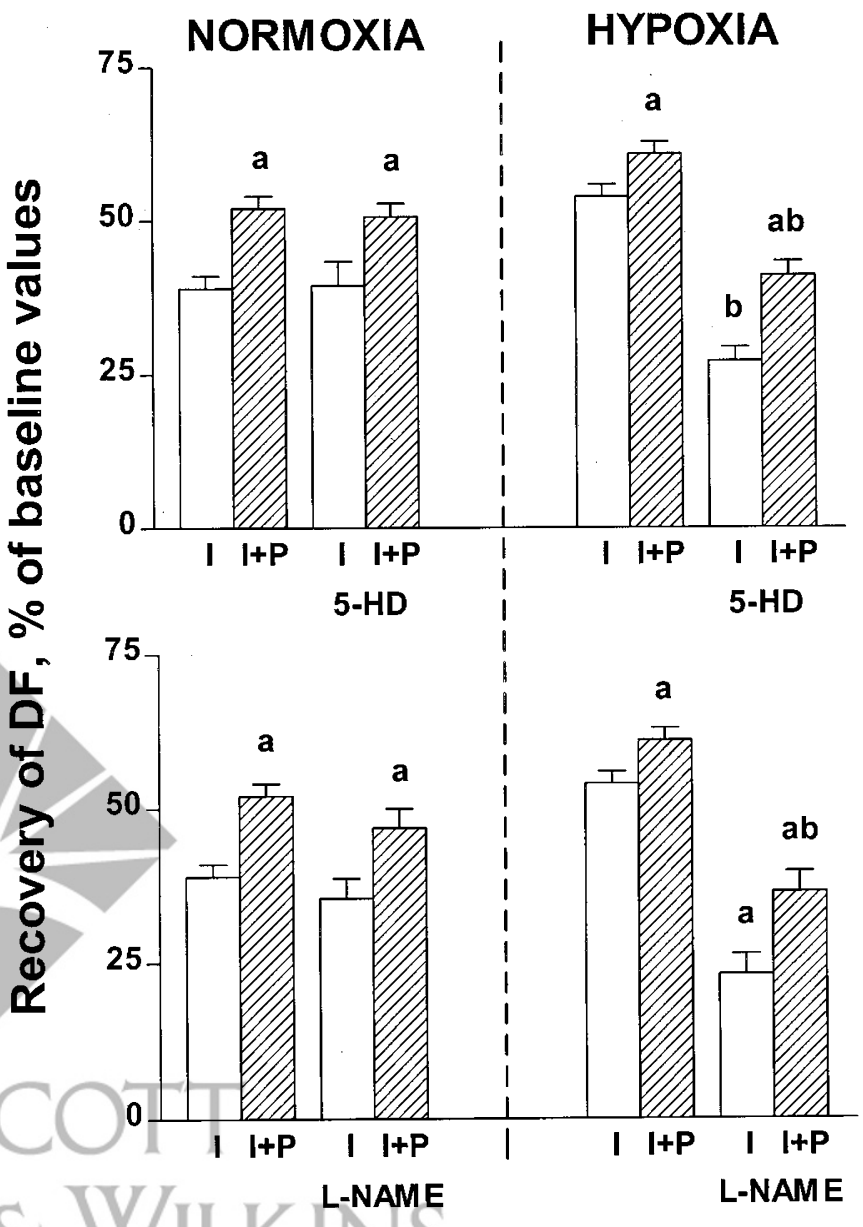

Figure 5. Effect of 5-HD and L-NAME on cardiac tolerance to ischemia, d 10. Cardiac tolerance to ischemia (expressed as the recovery of DF after ischemia) on d 10 of postnatal life in rats. Effect of ischemia (I) and ischemia and preconditioning $(\mathrm{I}+\mathrm{P})$ in normoxic and hypoxic animals perfused with or without 5-HD (upper panel) and with or without L-NAME (lower panel); ${ }^{\mathrm{a}}$ significant difference $(p<0.01)$ from the ischemic group (I); ${ }^{\mathrm{b}}$ from the groups perfused without 5-HD or L-NAME, respectively.

sial, probably due to different experimental protocols, particularly animal species, age, and end points of injury. In the only study on the immature heart, Baker et al. (11) have observed that, whereas the isolated rabbit myocardium (7- to 10-d-old), normoxic from birth, could be preconditioned (with recovery of the contractile function as the end point), isolated immature heart chronically hypoxic from birth could not be preconditioned, even when the number of occlusion periods was increased. They concluded that chronically hypoxic immature hearts are already protected and that additional cardioprotection by IP is not possible. Our study suggests that this is not valid for the neonatal rat heart, where the protective effect appears only by combination of both protective phenomena. Different results may be probably due to species differences, including the degree of maturation at birth and the time course of development during early stages of ontogeny. Moreover, differences in duration and degree of oxygen deprivation (permanent versus intermittent, $\mathrm{PO}_{2}$ level) have to be taken into consideration. There are, however, some other differences in 
Table 2. Coronary flow (measured at maximum recovery of DF during reperfusion) after ischemia (I) and ischemia with previous preconditioning $(I+P)$ in control hearts and hearts treated with L-NAME or 5-HD in normoxic or prenatally hypoxic 1-and 10-d-old rats

\begin{tabular}{|c|c|c|c|c|c|}
\hline \multirow[b]{2}{*}{ Rats } & \multirow[b]{2}{*}{ Age (d) } & & \multicolumn{3}{|c|}{ Percentage of baseline value } \\
\hline & & & Controls & L-NAME & $5-\mathrm{HD}$ \\
\hline \multirow[t]{3}{*}{ Normoxic } & 1 & I & $84.3 \pm 6.9$ & $33.0 \pm 7.8^{*}$ & $85.4 \pm 15.8$ \\
\hline & 10 & I & $86.7 \pm 5.7$ & $39.7 \pm 4.7^{*}$ & $71.3 \pm 7.6$ \\
\hline & & $\mathrm{I}+\mathrm{P}$ & $57.2 \pm 10.4$ & $35.6 \pm 4.4$ & $47.9 \pm 6.0$ \\
\hline \multirow[t]{3}{*}{ Hypoxic } & 1 & I & $68.2 \pm 8.9$ & $42.1 \pm 6.2 *$ & $47.7 \pm 5.0$ \\
\hline & & $\mathrm{I}+\mathrm{P}$ & $62.4 \pm 5.1$ & $50.2 \pm 5.9$ & $54.6 \pm 9.5$ \\
\hline & & $\mathrm{I}+\mathrm{P}$ & $79.9 \pm 6.2$ & $54.0 \pm 3.9^{*}$ & $65.0 \pm 4.3$ \\
\hline
\end{tabular}

* Significantly different $(p<0.05)$ from control hearts.

both protocols that might influence the final results e.g. the fact that in our experimental setup the sucklings were with their mothers even during their stay in the barochamber and our isolated hearts were electrically stimulated.

Tajima et al. (19) demonstrated in adult rats that the protective effect of preconditioning against subsequent postischemic contractile dysfunction is additive to that afforded by adaptation to chronic hypoxia. This may suggest that the two phenomena are independent and use different mechanisms. In contrast, our recent experiments in mature rats examining infarct size as the end point of injury have shown that combination of these two phenomena did not provide a better cardioprotective effect than preconditioning alone (20). We hypothesized, therefore, that these long- and short-term protective mechanisms might share the same signaling pathway or element. It seems, therefore, that protective mechanisms may differ during ontogenetic development, particularly in neonates (8).

To further analyze the above question, we have compared the possible involvement of mitochondrial $\mathrm{K}_{\mathrm{ATP}}$ channels in mechanisms of protection by adaptation to chronic hypoxia and IP in two developmental periods, differing considerably in their protective ability: postnatal $\mathrm{d} 1$ and 10 . We have found that the mitochondrial $\mathrm{K}_{\mathrm{ATP}}$ channel blocker 5-HD did not influence cardiac tolerance to ischemia in normoxic animals but significantly decreased this parameter in hypoxic rats, even below the normoxic values. The protective effect of IP was, however, preserved. It seems, therefore, that mitochondrial $\mathrm{K}_{\mathrm{ATP}}$ channels are involved in the protective mechanism of adaptation to chronic hypoxia but not in the mechanism of IP in this developmental period.

It is, however, unclear at present how the opening of mitochondrial $\mathrm{K}_{\mathrm{ATP}}$ channels may result in cardiac protection. It has been suggested that the main function of these channels is to control mitochondrial matrix volume, which, in turn, is thought to regulate electron transport and bioenergetics (29). This hypothesis is in agreement with the observation of Eells et al. (15) that the rate of ATP synthesis in immature heart adapted to chronic hypoxia was significantly greater than that in normoxic hearts. Mitochondrial $\mathrm{K}_{\mathrm{ATP}}$ channel activation may, therefore, be an essential component of the signal transduction pathway, calling for increased ATP production to support increased work of the heart or possibly to compensate for decreased oxygen availability.
Baker et al. (21) and Shi et al. (30) found that exposure of immature rabbit hearts to chronic hypoxia increased protein levels for endothelial NOS as well as release of nitrite, nitrate, and tissue cGMP content. Moreover, NO donors increased the recovery of postischemic function in normoxic hearts but not in hearts chronically hypoxic from birth. Conversely, NOS inhibitors abolished the cardioprotective effect of hypoxia in 7- and 10-d-old rabbits. We have observed that the effect of NOS inhibitor L-NAME was strictly age dependent: in 1-d-old hearts, it reduced postischemic recovery of DF in normoxic preconditioned and nonpreconditioned hearts and attenuated the protective effect of preconditioning in hypoxic animals; on the other hand, in 10-d-old hearts, L-NAME decreased significantly only the protective effect of adaptation to IHA; the protective effect of preconditioning remained unchanged. The difference between 1- and 10-d-old heart is not surprising inasmuch as significant developmental changes in NO sources (myocardium, vascular endothelium, endocardium) can be observed in rats during the first week of life (31). On the other hand, L-NAME significantly reduced CF in all treated hearts, suggesting that the effect on cardiomyocytes is not necessarily connected with the effect on endothelial cells. It may be assumed, therefore, that NO plays a role in the maintenance of high cardiac resistance to oxygen deprivation in normoxic newborns, and is involved also in the cardioprotective mechanism of adaptation to IHA in 10-d-old hearts.

And how might $\mathrm{NO}$ interact with mitochondrial $\mathrm{K}_{\mathrm{ATP}}$ channels? Baker et al. (21) proposed that NO leads to activation of $\mathrm{K}_{\mathrm{ATP}}$ channels via soluble guanylyl cyclase, causing accumulation of cGMP and activation of cGMP-dependent protein kinase. Moreover, $\mathrm{K}_{\text {ATP }}$ channels can be activated by lactate, the level of which is elevated in chronically hypoxic hearts (32). Recently, Sasaki et al. (33) demonstrated that exposure of myocytes to an NO donor directly activates mitochondrial $\mathrm{K}_{\mathrm{ATP}}$ channels.

We can conclude that prenatal exposure to IHA hypoxia failed to increase tolerance to ischemia in 1-d-old rat hearts; a cardioprotective effect appeared only after early postnatal exposure to IHA by the end of the first postnatal week. Combination of adaptation to IHA and IP, however, increased cardiac tolerance to ischemia even on postnatal $\mathrm{d} 1$, where both phenomena alone failed to improve resistance to oxygen deprivation. It seems likely that protective mechanisms may differ during ontogenetic development, particularly in neo- 
nates; mitochondrial $\mathrm{K}_{\mathrm{ATP}}$ channels and $\mathrm{NO}$ seem to be involved in the protective mechanism of adaptation to chronic hypoxia but not in the mechanism of IP in this developmental period.

Acknowledgment. The authors thank Mrs. Dědičová for statistical analysis.

\section{REFERENCES}

1. Oštádal B, Oštádalová I, Dhalla NS 1999 Development of cardiac sensitivity to oxygen deficiency: comparative and ontogenetic aspects. Physiol Rev 79:635-659

2. Hurtado A 1960 Some clinical aspects of life at high altitudes. Ann Intern Med 53:247-258

3. Poupa O, Krofta K, Procházka J, Turek Z 1966 Acclimatization to simulated high altitude and acute cardiac necrosis. Fed Proc 25:1243-1246

4. Murry CE, Jennings RB, Reimer KA 1986 Preconditioning with ischemia: a delay of lethal cell injury in ischemic myocardium. Circulation 74:1124-1136

5. Oštádal B, Koláŕ F, Pelouch V, Widimský J 1995 Ontogenetic differences in cardiopulmonary adaptation to chronic hypoxia. Physiol Res 44:45-51

6. Baker EJ, Boerboom LE, Olinger GN, Baker JE 1995 Tolerance of the developing heart to ischemia: impact of hypoxemia from birth. Am J Physiol 268:H1165-H1173

7. Oštádalová I, Koláŕ F, Oštádal B, Parratt JR 1996 Ischaemic preconditioning in neonatal rat hearts. J Physiol 491:P:6P-7P

8. Oštádalová I, Oštádal B, Koláŕ F, Parratt JR, Wilson S 1998 Tolerance to ischaemia and ischaemic preconditioning in neonatal rat heart. J Mol Cell Cardiol 30:857-865

9. Awad WI, Shattock MJ, Chambers DJ 1998 Ischemic preconditioning in immature myocardium. Circulation 98:II-206-II-213

10. Liu H, Cala PM, Anderson SE 1998 Ischemic preconditioning: effects on $\mathrm{pH}, \mathrm{Na}$ and $\mathrm{Ca}$ in newborn rabbit hearts during ischemic/reperfusion. J Mol Cell Cardiol 30:685 697

11. Baker JE, Holman P, Gross GJ 1999 Preconditioning in immature rabbit hearts. Role of $\mathrm{K}_{\mathrm{ATP}}$ channels. Circulation 99:1249-1254

12. Kolář F 1996 Cardioprotective effects of chronic hypoxia: relation to preconditioning In: Wainwright CL, Parratt JR (eds) Myocardial Preconditioning. Springer Verlag, Berlin, pp 261-275

13. Oštádal B, Kolář F 1999 Cardiac Ischemia: From Injury to Protection. Kluwer Academic Publishers, Boston, p 173

14. Asemu G, Papoušek F, Oštádal B, Kolář F 1999 Adaptation to high altitude hypoxia protects the rat heart against ischemia-induced arrhythmias. Involvement of mitochondrial $\mathrm{K}_{\mathrm{ATP}}$ channel. J Mol Cell Cardiol 31:1821-1831
15. Eells JT, Henry MH, Gross GJ, Baker JE 2000 Increased mitochondrial K(ATP) channel activity during chronic myocardial hypoxia: is cardioprotection mediated by improved bioenergetics? Circ Res 87:915-921

16. Baines CP, Wang L, Cohen MV, Downey JM 1999 Myocardial protection by insulin is dependent on phosphatidylinositol 3-kinase but not protein kinase $\mathrm{C}$ or $\mathrm{K}_{\mathrm{ATP}}$ channels in the isolated rabbit hearts. Basic Res Cardiol 94:188-198

17. Végh A, Komori S, Szekeres L, Parrat JR 1992 Antiarhytmic effects of preconditioning in anaesthetized dogs and rats. Cardiovasc Res 26:487-495

18. Baker JE, Contney SJ, Singh R, Kalyanaraman B, Gross GJ, Bosnjak ZJ 2001 Nitric oxyde activates the sarcolemmal $\mathrm{K}_{\mathrm{ATP}}$ channel in normoxic and chronically hypoxic hearts by a cyclic GMP-dependent mechanism. J Mol Cell Cardiol 33:331-341

19. Tajima M, Katayose D, Bessho M, Isoyama S 1994 Acute ischaemic preconditioning and chronic hypoxia independently increase myocardial tolerance to ischaemia. Cardiovasc Res 28:312-319

20. Neckář J, Papoušek F, Nováková O, Oštádal B, Kolář F 2002 Cardioprotective effects of chronic hypoxia and ischaemic preconditioning are not additive. Basic Res Cardiol 97:161-167

21. Baker JE, Boerboom LE, Olinger GN 1998 Age related changes in the ability of hypothermia and cardioplegia to protect ischemic rabbit myocardium. J Thorac Cardiovasc Surg 96:717-724

22. Oštádalová I, Kolář F, Oštádal B, Rohličèk V, Rohličèk J, Procházka J 1993 Early postnatal development of contractile performance and responsiveness to $\mathrm{Ca}^{2+}$, verapamil and ryanodine in the isolated rat heart. J Mol Cell Cardiol 25:733-740

23. Oštádalová I, Koláŕ F, Oštádal B 1995 Inotropic effect of low extracellular sodium on perfused perinatal rat heart. Can J Physiol Pharmacol 73:50-54

24. Lichtfield JB 1958 Blood pressure in infant rats. Physiol Zool 31:1-6

25. Zicha J, Kuneš J, Jelínek J 1986 Experimental hypertension in young and adult animals. Hypertension 8:1096-1104

26. Cave AC 1996 The protective effects of preconditioning on postischemic contractile function. In: Wainwright CL, Parratt JR (eds) Myocardial Preconditioning. RG

Landes Company, Austin, TX, pp 61-78

27. Riva E, Hearse DJ 1993 Age-dependent changes in myocardial susceptibility to ischemic injury. Cardioscience 4:85-92

28. Singer D 1999 Neonatal tolerance to hypoxia: a comparative-physiological approach. Comp Biochem Physiol A Mol Integr Physiol 123:221-234

29. Garlid KD 1996 Cation transport in mitochondria - the potassium cycle. Biochim Biophys Acta 1275:123-126

30. Shi Y, Pritchard KA, Holman P, Rafiee P, Griffith OW, Kalyanaraman B, Baker JE 2000 Chronic myocardial hypoxia increases nitric oxide synthase and decreases calveolin-3. Free Radic Biol Med 29:695-703

31. Oštádal B 2001 Nitric oxide and the developing heart. In: Gryglewski RJ, Minuz P (eds) Nitric Oxide: Basic Research and Clinical Applications. IOS Press, Amsterdam, pp $92-99$

32. Baker JE, Contney SJ, Gross GJ, Bosnjak ZJ $1997 \mathrm{~K}_{\text {ATT }}$ channel activation in a rabbit model of chronic myocarial hypoxia. J Mol Cell Cardiol 29:845-848

33. Sasaki N, Sato T, Ohler A, O'Rourke B, Marbán E 2000 Activation of mitochondrial ATP-dependent potassium channels by nitric oxide. Circulation 101:439-445 